Proceedings of the Edinburgh Mathematical Society (2002) 45, 285-300 (C)

DOI:10.1017/S0013091500000687 Printed in the United Kingdom

\title{
AN INTERNAL CHARACTERIZATION OF COMPLETE POSITIVITY FOR ELEMENTARY OPERATORS
}

\author{
RICHARD M. TIMONEY \\ School of Mathematics, Trinity College, Dublin 2, Ireland (richardt@maths.tcd.ie)
}

(Received 15 June 2000)

\begin{abstract}
Complete positivity of 'atomically extensible' bounded linear operators between $C^{*}$-algebras is characterized in terms of positivity of a bilinear form on certain finite-rank operators. In the case of an elementary operator on a $C^{*}$-algebra, the approach leads us to characterize $k$-positivity of the operator in terms of positivity of a quadratic form on a subset of the dual space of the algebra and in terms of a certain inequality involving factorial states of finite type I.

As an application we characterize those $C^{*}$-algebras where every $k$-positive elementary operator on the algebra is completely positive. They are either $k$-subhomogeneous or $k$-subhomogeneous by antiliminal. We also give a dual approach to the metric operator space introduced by Arveson.
\end{abstract}

Keywords: $C^{*}$-algebra; metric operator space; factorial state; subhomogeneous; antiliminal; atomic representation

AMS 2000 Mathematics subject classification: Primary 46L05

Secondary 47B47; 47B65

\section{Introduction}

For two $C^{*}$-algebras $A$ and $B$, a linear operator $T: A \rightarrow B$ is called positive if $T x \geqslant 0$ whenever $x \in A$ is positive. $T$ is called $k$-positive if the operator $T^{(k)}$ is positive, where $T^{(k)}$ acts on the $k \times k$ matrices $M_{k}(A)$ with entries in $A$, has values in $M_{k}(B)$ and is given by $T^{(k)}\left(x_{i j}\right)_{i, j}=\left(T\left(x_{i j}\right)\right)_{i, j}$. The operator $T$ is called completely positive if it is $k$-positive for all $k=1,2,3, \ldots$ This terminology goes back to Stinespring [10]. A general reference for the theory is $[\mathbf{8}]$, but we do not consider here the more general case of operators on operator systems. A notable recent development of the theory (for the case of normal operators on $\mathcal{B}(H)$ ) is the concept of a metric operator space introduced by Arveson in [4]. In Arveson's setting, our approach leads to a space which is dual to his metric operator space.

For a $C^{*}$-algebra $A$, an operator $T: A \rightarrow A$ is called an elementary operator if $T$ can be expressed in the form $T x=\sum_{j=1}^{\ell} a_{j} x b_{j}$ with $a_{j}$ and $b_{j}(1 \leqslant j \leqslant \ell$ ) in $A$. (More generally, one may allow $a_{j}$ and $b_{j}$ to be in the multiplier algebra $M(A)$ of $A$ (see [7]).) Such representations of $T$ may not be unique.

We denote by $\mathcal{B}(H)$ the bounded operators on a Hilbert space $H$, by $\mathcal{K}(H)$ the compact operators, by $\mathcal{F}_{k}(H)$ the operators of rank at most $k$ and by $\mathcal{F}(H)$ the finite-rank 
operators. In case $H$ is infinite dimensional, the dimension of $H$ is just $\infty$. We use $\langle\cdot, \cdot\rangle$ for the inner product on $H$.

Using results of $[\mathbf{6}]$ and $[\mathbf{1 1}]$, a form of the following result for the case $A=M_{n}(\mathbb{C})$ was proved in $[\mathbf{1 3}]$.

Theorem 1.1. Let $A$ be $\mathcal{B}(H)$ or $\mathcal{K}(H)$ for a Hilbert space $H$ of dimension $n \leqslant \infty$. Let $T: A \rightarrow A$ be an elementary operator of the form $T x=\sum_{j=1}^{\ell} a_{j} x b_{j}$ (where $a_{j}, b_{j} \in \mathcal{B}(H)$ for each $j$ ). Suppose $k \geqslant 1$. Then $T$ is $k$-positive if and only if the form $Q_{T}: \mathcal{F}(H) \rightarrow \mathbb{C}$ given by

$$
Q_{T}(x)=\sum_{j=1}^{\ell} \operatorname{tr}\left(a_{j} x^{*}\right) \operatorname{tr}\left(b_{j} x\right)
$$

satisfies $Q_{T}(x) \geqslant 0$ whenever $x \in \mathcal{F}_{k}(H)$.

To generalize this theorem to arbitrary $C^{*}$-algebras, we consider $Q_{T}$ as defined on the dual space $A^{*}$ and we introduce a notion of 'rank $k$ ' for elements of $A^{*}$.

Definition 1.2. For a $C^{*}$-algebra $A$ and $k \geqslant 1$, an element $\phi \in A^{*}$ of the dual space of $A$ is said to have rank at most $k$ and singleton support if there exists an irreducible *-representation $\pi: A \rightarrow \mathcal{B}\left(H_{\pi}\right)$ and an element $y_{\phi} \in \mathcal{F}_{k}\left(H_{\pi}\right)$ so that

$$
\phi(x)=\operatorname{tr}\left(\pi(x) y_{\phi}\right)
$$

for all $x \in A$. We denote the subset of $A^{*}$ made up of such elements by $\mathcal{F}_{k}^{s}(A)$ and we write $\mathcal{F}^{s}(A)=\bigcup_{k=1}^{\infty} \mathcal{F}_{k}^{s}(A)$.

Our main result is the following.

Theorem 1.3. Let $A$ be a $C^{*}$-algebra and let $T: A \rightarrow A$ be an elementary operator of the form $T x=\sum_{j=1}^{\ell} a_{j} x b_{j}$ (where $a_{j}, b_{j} \in M(A)$ for each $j$ ). Suppose $k \geqslant 1$. Then $T$ is $k$-positive if and only if the form $Q_{T}: \mathcal{F}_{k}^{s}(A) \rightarrow \mathbb{C}$, given by

$$
Q_{T}(\phi)=\sum_{j=1}^{\ell} \phi^{*}\left(a_{j}\right) \phi\left(b_{j}\right),
$$

satisfies $Q_{T}(\phi) \geqslant 0$ whenever $\phi \in \mathcal{F}_{k}^{s}(A)$.

$T$ is completely positive if and only if $Q_{T}(\phi) \geqslant 0$ for all $\phi \in \mathcal{F}^{s}(A)$.

The (reduced) atomic representation of $A$ (see $[\mathbf{9}, 4.3 .7]$ ) is used in our discussion. This atomic representation $\pi_{a}: A \rightarrow \mathcal{B}\left(H_{a}\right)$ is defined by choosing one irreducible representation $\pi_{t}: A \rightarrow \mathcal{B}\left(H_{t}\right)$ in each equivalence class $t \in \hat{A}$ ( $\hat{A}$ denotes the unitary equivalence classes of irreducible representations of $A$ ). Then $\pi_{a}=\bigoplus_{t \in \hat{A}} \pi_{t}$ is a faithful representation of $A$ on the Hilbert space $H_{a}=\bigoplus_{t \in \hat{A}} H_{t}$ and $\pi_{a}(A)$ is weak ${ }^{*}$-dense in $\mathcal{A}=\bigoplus_{t \in \hat{A}} \mathcal{B}\left(H_{t}\right)$ (equivalently, the double commutant of $\pi_{a}(A)$ is $\mathcal{A}$ ). The predual of $\mathcal{A}$ is the $\ell^{1}$-direct sum of the trace class operators $\mathcal{S}_{1}\left(H_{t}\right)$ on the spaces $H_{t}$. (The dual of each $\mathcal{S}_{1}\left(H_{t}\right)$ is $\mathcal{B}\left(H_{t}\right)$ via the trace duality.)

Our Definition 1.2 can be rephrased: an element $\phi$ of the dual $A^{*}$ is said to be of rank at most $k$ and singleton support if there exists $t \in \hat{A}$ and $y \in \mathcal{S}_{1}\left(H_{t}\right)$ of rank at most 
$k$ so that $\phi(x)=\operatorname{tr}\left(\pi_{t}(x) y\right)$. Consequently, $\phi$ corresponds to a certain kind of normal functional on $\mathcal{A}$. We refer to $t$ (or the representation $\pi_{t}$ ) as the support of $\phi$.

We can view the multiplier algebra $M(A)$ as a subalgebra of $A^{* *}$ or of the von Neumann algebra closure $\mathcal{A}$ of $\pi_{a}(A)$ (see [9, Corollary 3.12.5]). In this way the expression for $Q_{T}(\phi)$ given in the statement of the theorem makes sense for the situation where we allow $a_{j}, b_{j} \in M(A)$ and take $\phi \in \mathcal{F}_{k}^{s}(A)$.

In [5] it is observed that if a state $\phi$ is a factorial state (that is if the von Neumann algebra closure of the image $\pi_{\phi}(A)$ of $A$ in the corresponding representation is a factor) and if the commutant $\pi_{\phi}(A)^{\prime}$ is a factor of type $I_{k}(1 \leqslant k<\infty)$, then $\phi$ is a convex combination of $k$ equivalent pure states $\phi_{n}$ (all give the same $t \in \hat{A}$ ).

It is then easy to see that the factorial states $\phi$ for which $\pi_{\phi}(A)^{\prime}$ is a factor of type $I_{j}$ for some $j \leqslant k$ are contained in $\mathcal{F}_{k}^{s}(A)$. This set of factorial states is denoted $F_{k}(A)$ in $[5]$.

Recall that a $C^{*}$-algebra $A$ is called $k$-subhomogeneous if each irreducible representation of $A$ has dimension at most $k$. This means that the atomic representation maps into a direct sum of matrix algebras of sizes $k \times k$ or less. On the other hand, $A$ is called antiliminal if for each non-zero $x \in A$ there exists an irreducible representation $\pi_{t}$ of $A$ for which $\pi_{t}(x)$ is a non-compact operator.

With some small amount of work we can then recast Theorem 1.3 as follows.

Theorem 1.4. Let $A$ be a $C^{*}$-algebra and let $T: A \rightarrow A$ be an elementary operator of the form $T x=\sum_{j=1}^{\ell} a_{j} x b_{j}$ (where $a_{j}, b_{j} \in M(A)$ for each $j$ ). Suppose $k \geqslant 1$. Then $T$ is $k$-positive if and only if

$$
\sum_{j=1}^{\ell} \psi\left(a_{j} a^{*}\right) \psi\left(a b_{j}\right) \geqslant 0
$$

whenever $\psi \in F_{k}(A)$ and $a \in A$.

Using results of [5] we can deduce that all $k$-positive elementary operators on $A$ are completely positive if and only if $A$ is either $k$-subhomogeneous or $k$-subhomogeneous by antiliminal, thus extending the case $k=1$ dealt with in $[\mathbf{3}]$.

\section{The case of general $T$}

We aim for a version of Theorem 1.3 applicable to arbitrary bounded linear operators $T: A \rightarrow B$ between $C^{*}$-algebras $A$ and $B$. (By the device of considering the operator $: A \oplus B \rightarrow A \oplus B: a \oplus b \mapsto 0 \oplus T(a)$, it is often sufficient to deal with the case $A=B$.) However, we have not succeeded in this generality and we introduce a restriction on $T$.

We denote the reduced atomic representation of $B$ by $\pi_{a}=\bigoplus_{s \in \hat{B}} \pi_{s}$, with $\pi_{s}: B \rightarrow$ $\mathcal{B}\left(H_{s}\right)$. We also set $\mathcal{B}=\bigoplus_{s \in \hat{B}} \mathcal{B}\left(H_{s}\right)$, in analogy with the notation $\mathcal{A}$.

Definition 2.1. We call a bounded linear operator $T: A \rightarrow B$ between $C^{*}$-algebras atomically extensible if there exists a weak*-continuous $T_{a}: \mathcal{A} \rightarrow \mathcal{B}$ such that $\pi_{a} \circ T=$ $T_{a} \circ \pi_{a}$. 
In other words the following diagram commutes:

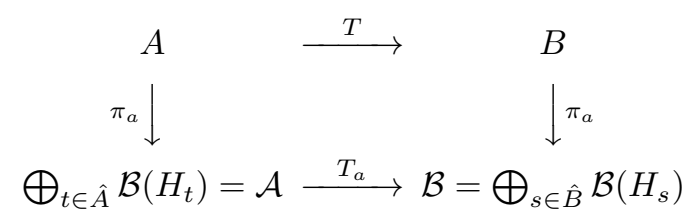

If $T$ is atomically extensible, then for each $t \in \hat{A}$ and $s \in \hat{B}$ we have a component mapping $T_{a, t s}: \mathcal{B}\left(H_{t}\right) \rightarrow \mathcal{B}\left(H_{s}\right)$ of $T_{a}$.

Note that each $T_{a, t s}$ is weak*-continuous and so also is

$$
T_{a, t s}^{(k)}: M_{k}\left(\mathcal{B}\left(H_{t}\right)\right)=\mathcal{B}\left(H_{t}^{k}\right) \rightarrow M_{k}\left(\mathcal{B}\left(H_{s}\right)\right) .
$$

(Here $H_{t}^{k}$ means the Hilbert space direct sum of $k$ copies of $H_{t}$.) Note also the following.

Lemma 2.2. For an atomically extensible bounded linear operator $T: A \rightarrow B$ between $C^{*}$-algebras, $k \geqslant 1$ and the above notation, $T$ is $k$-positive if and only if $T_{a, t s}$ is $k$-positive for each $t \in \hat{A}$ and $s \in \hat{B}$.

Lemma 2.3. For Hilbert spaces $H_{1}$ and $H_{2}$ and a weak*-continuous linear operator $T: \mathcal{B}\left(H_{1}\right) \rightarrow \mathcal{B}\left(H_{2}\right), T$ is positive if and only if $T$ maps each rank-one orthogonal projection in $\mathcal{B}\left(H_{1}\right)$ to a positive element of $\mathcal{B}\left(H_{2}\right)$.

Proof. The only if part is clear. Assuming that rank-one projections are mapped to positive elements, it follows easily that finite-rank positive elements in $\mathcal{B}\left(H_{1}\right)$ (which can all be expressed as positive linear combinations of rank-one projections) are mapped to positive elements of $\mathcal{B}\left(H_{2}\right)$.

Finally, if $x \in \mathcal{B}\left(H_{1}\right)$ is an arbitrary positive element, then we consider the net $\left(x_{P}\right)_{P}$ in $\mathcal{B}\left(H_{1}\right)$ indexed by the finite-rank self-adjoint projections $P \in \mathcal{B}\left(H_{1}\right)$ with $x_{P}=P x P$. This net converges to $x$ in the weak*-topology on $\mathcal{B}\left(H_{1}\right)$ and by weak*-continuity of $T$, $T\left(x_{P}\right) \rightarrow T(x)$ in the weak ${ }^{*}$-topology on $\mathcal{B}\left(H_{2}\right)$. Since $T\left(x_{P}\right) \geqslant 0$ for each $P$ it follows that $T(x) \geqslant 0$.

Notation. For $\xi \in H_{1}$ and $\eta \in H_{2}$ vectors in (possibly different) Hilbert spaces $H_{1}$ and $H_{2}$, we denote by $\xi^{*} \otimes \eta: H_{1} \rightarrow H_{2}$ the rank-one operator given by $\left(\xi^{*} \otimes \eta\right)(\alpha)=\langle\alpha, \xi\rangle \eta$ (for $\alpha \in H_{1}$ ). Every linear operator $W: H_{1} \rightarrow H_{2}$ of rank $k$ can be expressed in the form

$$
W=\sum_{j=1}^{k} \xi_{j}^{*} \otimes \eta_{j},
$$

with $\xi_{j} \in H_{1}$ and $\eta_{j} \in H_{2}(1 \leqslant j \leqslant k)$. In this way we can establish an isomorphism between the algebraic tensor product $\bar{H}_{1} \otimes H_{2}$ and the space, denoted by $\mathcal{F}\left(H_{1}, H_{2}\right)$, of finite-rank operators from $H_{1}$ to $H_{2}$. (Here $\bar{H}_{1}$ denotes the conjugate of $H_{1}$.) We denote by $\mathcal{F}_{k}\left(H_{1}, H_{2}\right)$ the subset of $\mathcal{F}\left(H_{1}, H_{2}\right)$ consisting of operators of rank at most $k$. Each $W \in \mathcal{F}_{k^{\prime}}\left(H_{1}, H_{2}\right)$ can be represented in the form (2.1) with linearly independent $\xi_{j} \in H_{1}$, linearly independent $\eta_{j} \in H_{2}$ and $k \leqslant k^{\prime}$. 
Proposition 2.4. Let $H_{1}$ and $H_{2}$ be Hilbert spaces and $T: \mathcal{B}\left(H_{1}\right) \rightarrow \mathcal{B}\left(H_{2}\right)$ a weak*-continuous linear operator. Then there is a well-defined form

$$
\mathcal{Q}_{T}: \mathcal{F}\left(H_{1}, H_{2}\right) \rightarrow \mathbb{C}
$$

given by

$$
\mathcal{Q}_{T}(W)=\sum_{i, j=1}^{k}\left\langle T\left(\xi_{i}^{*} \otimes \xi_{j}\right) \eta_{i}, \eta_{j}\right\rangle
$$

for $W$ as in (2.1). This form satisfies $\mathcal{Q}_{T}(\lambda W)=|\lambda|^{2} \mathcal{Q}_{T}(W)$ for $\lambda \in \mathbb{C}, W \in \mathcal{F}\left(H_{1}, H_{2}\right)$.

Moreover, if $k \geqslant 1$, then $T$ is $k$-positive if and only if $\mathcal{Q}_{T}(W) \geqslant 0$ for all $W \in$ $\mathcal{F}_{k}\left(H_{1}, H_{2}\right)$.

Proof. We can check that $\mathcal{Q}_{T}$ is well defined by considering the multilinear map

$$
R_{T}: \bar{H}_{1} \times H_{2} \times H_{1} \times \bar{H}_{2} \rightarrow \mathbb{C}
$$

given by

$$
R_{T}\left(\xi_{1}, \eta_{1}, \xi_{2}, \eta_{2}\right)=\left\langle T\left(\xi_{1}^{*} \otimes \xi_{2}\right) \eta_{1}, \eta_{2}\right\rangle,
$$

which therefore gives rise to a bilinear form : $\left(\bar{H}_{1} \otimes H_{2}\right) \times \overline{\left(\bar{H}_{1} \otimes H_{2}\right)} \rightarrow \mathbb{C}$. Restricting this to the 'diagonal' and taking into account the identification (2.1) between $\bar{H}_{1} \otimes H_{2}$ and $\mathcal{F}\left(H_{1}, H_{2}\right)$ gives $\mathcal{Q}_{T}$.

Applying Lemma 2.3 to

$$
T^{(k)}: M_{k}\left(\mathcal{B}\left(H_{1}\right)\right)=\mathcal{B}\left(H_{1}^{k}\right) \rightarrow M_{k}\left(\mathcal{B}\left(H_{2}\right)\right)=\mathcal{B}\left(H_{2}^{k}\right),
$$

we see that $T$ is $k$-positive if and only if $T^{(k)}\left(\xi^{*} \otimes \xi\right) \geqslant 0$ in $\mathcal{B}\left(H_{2}^{k}\right)$ whenever $\xi \in H_{1}^{k}$. This is in turn equivalent to $\left\langle T^{(k)}\left(\xi^{*} \otimes \xi\right) \eta, \eta\right\rangle \geqslant 0$ for all $\xi \in H_{1}^{k}$ and $\eta \in H_{2}^{k}$. Considering

$$
\xi=\left(\begin{array}{c}
\xi_{1} \\
\xi_{2} \\
\vdots \\
\xi_{k}
\end{array}\right), \quad \eta=\left(\begin{array}{c}
\eta_{1} \\
\eta_{2} \\
\vdots \\
\eta_{k}
\end{array}\right) \quad\left(\xi_{i} \in H_{1}, \eta_{i} \in H_{2}, 1 \leqslant i \leqslant k\right)
$$

we can write $\xi^{*} \otimes \xi \in M_{k}\left(\mathcal{B}\left(H_{1}\right)\right)$ as follows:

$$
\xi^{*} \otimes \xi=\left(\xi_{j}^{*} \otimes \xi_{i}\right)_{i, j=1}^{k} .
$$

Our condition for $k$-positivity then becomes

$$
\sum_{i, j=1}^{k}\left\langle T\left(\xi_{j}^{*} \otimes \xi_{i}\right) \eta_{j}, \eta_{i}\right\rangle \geqslant 0
$$

and the result follows. 
For two $C^{*}$-algebras $A$ and $B$, together with choices made of representations for their atomic representations, we define associated classes of finite-rank operators by $\mathcal{F}_{k}(A, B)=\bigcup_{t \in \hat{A}, s \in \hat{B}} \mathcal{F}_{k}\left(H_{t}, H_{s}\right)$ (disjoint union apart from 0 ), $\mathcal{F}(A, B)=$ $\bigcup_{k=1}^{\infty} \mathcal{F}_{k}(A, B)$.

For an atomically extensible bounded linear operator $T: A \rightarrow B$ we define $\mathcal{Q}_{T}$ on $\mathcal{F}(A, B)$ by

$$
\mathcal{Q}_{T}(W)=\mathcal{Q}_{T_{a, t s}}(W) \text { for } W \in \mathcal{F}\left(H_{t}, H_{s}\right) .
$$

Theorem 2.5. For an atomically extensible bounded linear operator $T: A \rightarrow B$ between $C^{*}$-algebras $A$ and $B$, and $k \geqslant 1, T$ is $k$-positive if and only if $\mathcal{Q}_{T}(W) \geqslant 0$ for all $W \in \mathcal{F}_{k}(A, B)$.

Proof. The proof follows by Lemma 2.2 and Proposition 2.4.

From this result we can immediately deduce (for atomically extensible operators only, however) the well-known result that if either $A$ or $B$ is abelian, then every positive linear operator $T: A \rightarrow B$ is completely positive. More generally, if either $A$ or $B$ is $k$-subhomogeneous $(k \geqslant 1)$, then every $k$-positive atomically extensible linear operator $T: A \rightarrow B$ is completely positive.

Example 2.6. Elementary operators are atomically extensible.

As noted above (in $\S 1$ ), we can view the multiplier algebra $M(A)$ as a subalgebra of $\mathcal{A}$. In this way the formula for an elementary operator $T(x)=\sum_{j=1}^{\ell} a_{j} x b_{j}$ on $A$ can be used to give $T_{a}$.

Example 2.7. For the case $A=C(K)$, with $K$ a compact Hausdorff space, and $\tau: K \rightarrow K$ continuous, the composition operator $T: A \rightarrow A$ given by $(T f)(x)=f(\tau(x))$ (for $f \in C(K), x \in K$ ) is atomically extensible (but not elementary if $\tau$ is not the identity). In this case, $\mathcal{A}=\ell^{\infty}(K)$ is the space of all bounded functions on $K$ (the dual of $\left.\ell^{1}(K)\right)$. Here $T_{a}$ is given by the same formula as $T$.

Example 2.8. For the case $A=C[0,1]$, the operator $T: A \rightarrow A$ given by $(T f)(x)=$ $\int_{0}^{1} f(t) \mathrm{d} t$ is not atomically extensible. In this situation, for the element $\delta_{t_{0}} \in \mathcal{A}=$ $\ell^{\infty}([0,1])$ given by $\delta_{t_{0}}(t)=0$ if $t \neq t_{0}$ and $\delta_{t_{0}}\left(t_{0}\right)=1$, we can find a sequence of elements of $C[0,1]$ which tend pointwise to $\delta_{t_{0}}$ and are bounded by 1 . It follows that this sequence tends to $\delta_{t_{0}}$ in the weak*-topology of $\ell^{\infty}([0,1])$. Also $T$ tends to zero along such a sequence. Hence any atomic extension $T_{a}$ would have to have $T_{a}\left(\delta_{t_{0}}\right)=0$ for all $t_{0} \in[0,1]$. As the linear span of the $\delta_{t_{0}}$ is weak*-dense in $\ell^{\infty}([0,1])$, it would follow that $T_{a}=0$.

Example 2.9. If $A=c_{0}$ or $A=\mathcal{K}(H)$ or $A$ is a $c_{0}$ direct sum of various $\mathcal{K}\left(H_{i}\right)$, then $\mathcal{A}$ is the double dual of $A$. So, if $A$ and $B$ are both of this type, then every $T: A \rightarrow B$ is atomically extensible (with $T_{a}$ the double transpose of $T$ ).

Remark 2.10. It is straightforward to check that $\mathcal{Q}_{T}$ is real-valued if $T$ sends hermitian elements of $A$ to hermitians in $B$, or, equivalently, if $T\left(x^{*}\right)=(T x)^{*}$ for $x \in A$. In 
that case, taking $W \in \mathcal{F}_{k}\left(H_{s}, H_{t}\right) \subset \mathcal{F}_{k}(A, B)$ expressed in the form (2.1), we have

$$
\begin{aligned}
\overline{\mathcal{Q}_{T}(W)} & =\sum_{i, j=1}^{k}\left\langle\eta_{j}, T\left(\xi_{i}^{*} \otimes \xi_{j}\right) \eta_{i}\right\rangle \\
& =\sum_{i, j=1}^{k}\left\langle\eta_{j}, T\left(\left(\xi_{j}^{*} \otimes \xi_{i}\right)^{*}\right) \eta_{i}\right\rangle \\
& =\sum_{i, j=1}^{k}\left\langle\eta_{j},\left(T\left(\xi_{j}^{*} \otimes \xi_{i}\right)\right)^{*} \eta_{i}\right\rangle \\
& =\sum_{i, j=1}^{k}\left\langle T\left(\xi_{j}^{*} \otimes \xi_{i}\right) \eta_{j}, \eta_{i}\right\rangle \\
& =\mathcal{Q}_{T}(W) .
\end{aligned}
$$

\section{The case $A=\mathcal{B}(H)$}

In this section, we prove Theorem 1.1 and we establish a connection with the metric operator space of Arveson [4].

Lemma 3.1. Let $A=\mathcal{B}(H), T: A \rightarrow A$ an elementary operator and $Q_{T}$ as in Theorem 1.1. For $x \in \mathcal{F}_{k}(H)$, we have

$$
Q_{T}(x)=\mathcal{Q}_{T}(x)
$$

(for $\mathcal{Q}_{T}$ as in Proposition 2.4 with $H_{1}=H_{2}=H$ ).

Proof. Recall that each element $x \in \mathcal{F}_{k}(H)$ can be represented in the form $x=$ $\sum_{r=1}^{k^{\prime}} \xi_{r}^{*} \otimes \eta_{r}$, with $\xi_{r}, \eta_{r} \in H$ for $1 \leqslant r \leqslant k^{\prime} \leqslant k$. Any such $x$ has rank at most $k^{\prime}$, and for $x$ of rank $k^{\prime}$ we can choose $\xi_{1}, \xi_{2}, \ldots, \xi_{k^{\prime}}$ to be an orthonormal basis for the orthogonal complement of the kernel of $x$ and $\eta_{r}=x\left(\xi_{r}\right)$.

To compute traces, it is convenient to assume that the $\xi_{r}$ are orthonormal and to compute via an orthonormal basis for $H$ made up of $\xi_{1}, \xi_{2}, \ldots, \xi_{k^{\prime}}$ with additional vectors added. Then

$$
\begin{aligned}
\operatorname{tr}(x) & =\sum_{r=1}^{k^{\prime}}\left\langle x\left(\xi_{r}\right), \xi_{r}\right\rangle, \\
\operatorname{tr}\left(b_{j} x\right) & =\sum_{r=1}^{k^{\prime}}\left\langle b_{j} x\left(\xi_{r}\right), \xi_{r}\right\rangle=\sum_{r=1}^{k^{\prime}}\left\langle b_{j} \eta_{r}, \xi_{r}\right\rangle
\end{aligned}
$$

and

$$
\operatorname{tr}\left(a_{j} x^{*}\right)=\overline{\operatorname{tr}\left(a_{j}^{*} x\right)}=\overline{\sum_{r=1}^{k^{\prime}}\left\langle a_{j}^{*} \eta_{r}, \xi_{r}\right\rangle}=\sum_{r=1}^{k^{\prime}}\left\langle a_{j} \xi_{r}, \eta_{r}\right\rangle
$$


Thus

$$
Q_{T}(x)=\sum_{j=1}^{\ell} \sum_{s=1}^{k^{\prime}}\left\langle a_{j} \xi_{s}, \eta_{s}\right\rangle \sum_{r=1}^{k^{\prime}}\left\langle b_{j} \eta_{r}, \xi_{r}\right\rangle=\sum_{j=1}^{\ell} \sum_{r, s=1}^{k^{\prime}}\left\langle a_{j} \xi_{s}, \eta_{s}\right\rangle\left\langle b_{j} \eta_{r}, \xi_{r}\right\rangle .
$$

But also

$$
\begin{aligned}
\mathcal{Q}_{T}(x) & =\sum_{r, s=1}^{k^{\prime}}\left\langle T\left(\xi_{r}^{*} \otimes \xi_{s}\right) \eta_{r}, \eta_{s}\right\rangle \\
& =\sum_{r, s=1}^{k^{\prime}} \sum_{j=1}^{\ell}\left\langle a_{j}\left\langle b_{j} \eta_{r}, \xi_{r}\right\rangle \xi_{s}, \eta_{s}\right\rangle \\
& =\sum_{r, s=1}^{k^{\prime}} \sum_{j=1}^{\ell}\left\langle a_{j} \xi_{s}, \eta_{s}\right\rangle\left\langle b_{j} \eta_{r}, \xi_{r}\right\rangle .
\end{aligned}
$$

Proof of Theorem 1.1. The case $A=\mathcal{K}(H)$ can be reduced to the case $A=\mathcal{B}(H)$ by a weak*-continuity argument.

The result follows from Lemma 3.1 and Proposition 2.4.

Remark 3.2. Natural questions are whether the form $Q_{T}$ introduced in Theorem 1.1 depends on the representation of $T$ as an elementary operator and whether $\mathcal{F}(H)$ is the natural domain for $Q_{T}$.

The independence of $Q_{T}$ from the representation of $T$ follows from Lemma 3.1.

For the question of the natural domain of $Q_{T}$, we consider the more general case of weak ${ }^{*}$-continuous operators $T$ and $\mathcal{Q}_{T}$.

Proposition 3.3. For a weak ${ }^{*}$-continuous bounded linear operator $T: \mathcal{B}(H) \rightarrow \mathcal{B}(H)$ and $W \in \mathcal{F}_{k}(H)$, we have

$$
\left|\mathcal{Q}_{T}(W)\right| \leqslant\left\|T^{(k)}\right\|\|W\|_{1}^{2}
$$

Proof. From the proof of Proposition 2.4 we can see that

$$
\left|\mathcal{Q}_{T}(W)\right|=\left|\left\langle T^{(k)}\left(\xi^{*} \otimes \xi\right) \eta, \eta\right\rangle\right| \leqslant\left\|T^{(k)}\right\|\left\|\xi^{*} \otimes \xi\right\|\|\eta\|^{2}
$$

if $W$ is as in $(2.1), \xi=\left(\xi_{1}, \ldots, \xi_{k}\right), \eta=\left(\eta_{1}, \ldots, \eta_{k}\right)$;

By polar decomposition we can rewrite $W=\sum_{j=1}^{k^{\prime}} \lambda_{j}^{2}\left(\xi_{j}^{\prime}\right)^{*} \otimes \eta_{j}^{\prime}$ with $0 \leqslant k^{\prime} \leqslant k$ and orthonormal $\xi_{j}^{\prime}$, orthonormal $\eta_{j}^{\prime}$ and $\lambda_{j}>0$. We can then assume $\xi_{j}=\lambda_{j} \xi_{j}^{\prime}$ and $\eta_{j}=\lambda_{j} \eta_{j}^{\prime}$ for $1 \leqslant j \leqslant k^{\prime}$. Now

$$
\left\|\xi^{*} \otimes \xi\right\|=\|\xi\|^{2}=\sum_{j} \lambda_{j}^{2}, \quad\|\eta\|=\sqrt{\sum_{j} \lambda_{j}^{2}}, \quad\|W\|_{1}=\sum_{j} \lambda_{j}^{2} .
$$


It follows that for completely bounded $T$ (that is where $\sup _{k}\left\|T^{(k)}\right\|<\infty$ ), we can make sense of $Q_{T}(x)=\mathcal{Q}_{T}(x)$ for any trace class $x \in \mathcal{S}_{1}(H)$ and the values of the extension of $Q_{T}$ will all be limits of the values on $\mathcal{F}(H)$.

If $T: \mathcal{B}(H) \rightarrow \mathcal{B}(H)$ is completely positive (thus automatically completely bounded) and weak*-continuous, then $\mathcal{Q}_{T} \geqslant 0$ and $\mathcal{Q}_{T}$ is related to a semi-inner product on $\mathcal{F}(H)$ or $\mathcal{S}_{1}(H) . \mathcal{Q}_{T}$ can be extended by continuity to the corresponding completion of (a quotient of) $\mathcal{F}(H)$ (or $\mathcal{S}_{1}(H)$ ). We denote this completion by $\mathcal{F}_{T}(H)$ and note that it will be a Hilbert space with respect to the continuous extension of the seminorm $\sqrt{\mathcal{Q}_{T}}$ on $\mathcal{F}(H)$. On $\mathcal{F}(H)$ the corresponding semi-inner product is

$$
\left\langle W, W^{\prime}\right\rangle_{T}=\sum_{i, j}\left\langle T\left(\xi_{i}^{*} \otimes \xi_{j}^{\prime}\right) \eta_{i}, \eta_{j}^{\prime}\right\rangle
$$

for $W=\sum_{i} \xi_{i}^{*} \otimes \eta_{i}, W^{\prime}=\sum_{j}\left(\xi_{j}^{\prime}\right)^{*} \otimes \eta_{j}^{\prime}$.

For $T(x)=\sum_{j=1}^{\ell} a_{j} x b_{j}$ elementary and completely positive, each of the operators $a_{j}$, $b_{j}(1 \leqslant j \leqslant \ell)$ defining $T$ defines a subspace such as $\left\{x \in \mathcal{F}(H): \operatorname{tr}\left(b_{j} x\right)=0\right\}$ of codimension one. Thus the kernel of $\mathcal{Q}_{T}=Q_{T}$ on $\mathcal{F}(H)$ will have finite codimension and the completion $\mathcal{F}_{T}(H)$ will in fact be a finite-dimensional quotient of $\mathcal{F}(H)$.

We can relate $\mathcal{F}_{T}(H)$ to the metric operator space of $T$ defined by Arveson [4] (for weak ${ }^{*}$-continuous completely positive $T: \mathcal{B}(H) \rightarrow \mathcal{B}(H)$ ). For $a \in \mathcal{B}(H)$ define $\Omega_{a}$ : $\mathcal{B}(H) \rightarrow \mathcal{B}(H)$ by $\Omega_{a}(x)=a x a^{*}$. The metric operator space of $T$ is (as a set) the set $\mathcal{E}_{T}$ of all $a \in \mathcal{B}(H)$ such that there is a positive $\lambda$ so that $\lambda T-\Omega_{a}$ is completely positive.

Restricting to the case of separable $H$, an inner product is defined on $\mathcal{E}_{T}$ as follows [4]. Via a minimal Stinespring representation of $T$, it is shown [4] that $T$ can be expressed as a finite or infinite sum $T x=\sum_{r} a_{r} x a_{r}^{*}$ where $a_{r} \in \mathcal{B}(H)$ satisfy $\sum_{r}\left\|a_{r} \xi\right\|^{2}<\infty$ for each $\xi \in H$ and an $\ell^{2}$ linear independence condition:

$$
\left(\alpha_{1}, \alpha_{2}, \ldots\right) \in \ell^{2}, \quad \sum_{r} \alpha_{r} a_{r}=0 \Rightarrow \alpha_{1}=\alpha_{2}=\cdots=0 .
$$

In this setting it is shown in [4] that $a \in \mathcal{E}_{T} \Longleftrightarrow a=\sum_{r} \alpha_{r} a_{r}$ for some $\left(\alpha_{r}\right)_{r} \in \ell^{2}$. The Hilbert space structure given to $\mathcal{E}_{T}$ in [4] makes $\left\{a_{r}: r \geqslant 1\right\}$ an orthonormal basis:

$$
\|a\|_{\mathcal{E}_{T}}=\sqrt{\sum_{r}\left|\alpha_{r}\right|^{2}} .
$$

Proposition 3.4. If $T: \mathcal{B}(H) \rightarrow \mathcal{B}(H)$ is weak ${ }^{*}$-continuous and completely positive, then the dual of $\mathcal{F}_{T}(H)$ may be identified with $\mathcal{E}_{T}$ as a set via trace duality.

Every continuous linear functional $\alpha$ on $\mathcal{F}_{T}(H)$ can be realized by a unique $a \in \mathcal{E}_{T}$ via

$$
\alpha(x)=\operatorname{tr}\left(a^{*} x\right) \quad \text { for } x \in \mathcal{F}(H),
$$

and each $a \in \mathcal{E}_{T}$ defines a continuous linear functional on $\mathcal{F}_{T}(H)$ in this way.

In the case of separable $H$, the identification of the dual of $\left(\mathcal{F}_{T}(H), \sqrt{\mathcal{Q}_{T}}\right)$ and $\left(\mathcal{E}_{T},\|\cdot\|_{\mathcal{E}_{T}}\right)$ is isometric and conjugate linear. 
Proof. If $a \in \mathcal{E}_{T}$ and $\lambda>0$ is such that $\lambda T-\Omega_{a}$ is completely positive, then $\mathcal{Q}_{\lambda T-\Omega_{a}}=$ $\lambda \mathcal{Q}_{T}-\mathcal{Q}_{\Omega_{a}}$ is non-negative on $\mathcal{F}(H)$ by Proposition 2.4 (with $H_{1}=H_{2}=H$ ). By Lemma 3.1,

$$
\mathcal{Q}_{\Omega_{a}}(x)=Q_{\Omega_{a}}\left(x^{*}\right)=\operatorname{tr}\left(a x^{*}\right) \operatorname{tr}\left(a^{*} x\right)=\left|\operatorname{tr}\left(a^{*} x\right)\right|^{2}
$$

for $x \in \mathcal{F}(H)$. Thus the non-negativity translates into

$$
\left|\operatorname{tr}\left(a^{*} x\right)\right| \leqslant \sqrt{\lambda} \sqrt{\mathcal{Q}_{T}(x)} .
$$

So the linear map $x \mapsto \operatorname{tr}\left(a^{*} x\right)$ on $\mathcal{F}(H)$ has a continuous extension to $\mathcal{F}_{T}(H)$.

Conversely, given a continuous linear functional $\alpha$ on $\mathcal{F}_{T}(H)$, we can apply it to rankone operators $\xi^{*} \otimes \eta \in \mathcal{F}_{1}(H) \subset \mathcal{F}(H)$ (or to their images in the quotient of $\mathcal{F}(H)$ modulo the kernel of $\left.\mathcal{Q}_{T}\right)$. We have

$$
\begin{aligned}
\left|\alpha\left(\xi^{*} \otimes \eta\right)\right| & \leqslant\|\alpha\| \sqrt{\mathcal{Q}_{T}\left(\xi^{*} \otimes \eta\right)} \\
& =\|\alpha\| \sqrt{\left\langle T\left(\xi^{*} \otimes \xi\right) \eta, \eta\right\rangle} \\
& \leqslant\|\alpha\| \sqrt{\|T\|}\|\xi\|\|\eta\|
\end{aligned}
$$

(using Proposition 3.3). It follows from the Riesz representation theorem applied to $H$ (with $\xi$ held fixed) that there is a unique element $a(\xi) \in H$ such that

$$
\alpha\left(\xi^{*} \otimes \eta\right)=\langle\eta, a(\xi)\rangle \quad(\eta \in H),
$$

and it is straightforward to check that the resulting $a: H \rightarrow H$ is linear. Also $\|a\| \leqslant$ $\|\alpha\| \sqrt{\|T\|}$ and so $a \in \mathcal{B}(H)$. Moreover,

$$
\alpha\left(\xi^{*} \otimes \eta\right)=\langle\eta, a(\xi)\rangle=\operatorname{tr}\left(a^{*}\left(\xi^{*} \otimes \eta\right)\right) .
$$

Hence $\alpha(x)=\operatorname{tr}\left(a^{*} x\right)$ for all $x \in \mathcal{F}(H)$ by linearity. The inequality

$$
|\alpha(x)| \leqslant\|\alpha\| \sqrt{\mathcal{Q}_{T}(x)} \quad(x \in \mathcal{F}(H))
$$

can be used to show that $a \in \mathcal{E}_{T}$ by reversing the steps at the beginning of the proof. By continuity, $\alpha$ must be the continuous extension of $x \mapsto \operatorname{tr}\left(a^{*} x\right)$ from $\mathcal{F}(H)$ to $\mathcal{F}_{T}(H)$.

It is easy to see that only one $a$ can have identical values of $\langle\eta, a(\xi)\rangle$ for $\xi, \eta \in H$ and so there is a unique $a$ for each $\alpha$.

Turning now to the isometry part, we suppose that $H$ is separable and consider $T$ represented as $T x=\sum_{r} a_{r} x a_{r}^{*}$ with the various restrictions on the choice of the $a_{r}$ introduced before the statement of the proposition.

For finite rank $W=\sum_{i} \xi_{i}^{*} \otimes \eta_{i}$, we have

$$
\begin{aligned}
\mathcal{Q}_{T}(W) & =\sum_{i, j}\left\langle T\left(\xi_{i}^{*} \otimes \xi_{j}\right) \eta_{i}, \eta_{j}\right\rangle \\
& =\sum_{i, j} \sum_{r}\left\langle a_{r}\left(\xi_{i}^{*} \otimes \xi_{j}\right) a_{r}^{*} \eta_{i}, \eta_{j}\right\rangle \\
& =\sum_{r} \mathcal{Q}_{\Omega_{a_{r}}}(W) \\
& =\sum_{r}\left|\operatorname{tr}\left(a_{r}^{*} W\right)\right|^{2} .
\end{aligned}
$$


For $a=\sum_{r} \alpha_{r} a_{r} \in \mathcal{E}_{T}$, it follows from the Cauchy-Schwarz inequality that

$$
\begin{aligned}
\left|\operatorname{tr}\left(a^{*} W\right)\right| & =\left|\sum_{r} \bar{\alpha}_{r} \operatorname{tr}\left(a_{r}^{*} W\right)\right| \\
& \leqslant \sqrt{\sum_{r}\left|\alpha_{r}\right|^{2}} \sqrt{\sum_{r}\left|\operatorname{tr}\left(a_{r}^{*} W\right)\right|^{2}} \\
& =\|a\|_{\mathcal{E}_{T}} \sqrt{\mathcal{Q}_{T}(W)} .
\end{aligned}
$$

Thus the norm of $a$ in the dual of $\mathcal{F}_{T}(H)$ is at most $\|a\|_{\mathcal{E}_{T}}$.

For the reverse inequality we rely on the $\ell^{2}$ linear independence condition (3.1), which we rephrase to say that if $\left(\alpha_{1}, \alpha_{2}, \ldots\right) \in \ell^{2}$ is non-zero, then there exist vectors $\xi, \eta \in H$ with

$$
\sum_{r} \bar{\alpha}_{r}\left\langle\eta, a_{r} \xi\right\rangle=\sum_{r} \bar{\alpha}_{r} \operatorname{tr}\left(a_{r}^{*}\left(\xi^{*} \otimes \eta\right)\right) \neq 0 .
$$

Rephrasing again, and using the Hahn-Banach Theorem, it says that the linear span of all the sequences $\left(\operatorname{tr}\left(a_{r}^{*}\left(\xi^{*} \otimes \eta\right)\right)\right)_{r}$ is dense in $\ell^{2}$. This linear span consists of

$$
\left\{\left(\operatorname{tr}\left(a_{r}^{*} W\right)\right)_{r}: W \in \mathcal{F}(H)\right\}
$$

Using this we can see that given $a=\sum_{r} \alpha_{r} a_{r} \in \mathcal{E}_{T}$, we can pick $W \in \mathcal{F}(H)$ with $\mathcal{Q}_{T}(W)=\sum_{r}\left|\operatorname{tr}\left(a_{r}^{*} W\right)\right|^{2}=1$ and

$$
\operatorname{tr}\left(a^{*} W\right)=\sum_{r} \bar{\alpha}_{r} \operatorname{tr}\left(a_{r}^{*} W\right)
$$

arbitrarily close to $\sqrt{\sum_{r}\left|\alpha_{r}\right|^{2}}=\|a\|_{\mathcal{E}_{T}}$. Thus we establish the desired reverse inequality.

Remark 3.5. In view of the argument above leading to (3.2) and the above isometry, we can see that for $a \in \mathcal{E}_{T}$, the smallest $\lambda>0$ such that $\lambda T-\Omega_{a}$ is completely positive is $\lambda=\|a\|_{\mathcal{E}_{T}}^{2}$. (This is also shown in [4, p. 560].)

Our proof shows that the dual norm of $\left(\mathcal{F}_{T}(H), \sqrt{\mathcal{Q}_{T}}\right)$ is the norm on $\mathcal{E}_{T}$ given by $\|a\|_{\mathcal{E}_{T}}^{2}=\inf \left\{\lambda \geqslant 0: \lambda T-\Omega_{a}\right.$ is completely positive $\}$, and establishes that this gives a Hilbertian norm on $\mathcal{E}_{T}$ even without a separability restriction on $H$ (assumed in [4]).

\section{The case of general $A$}

We now prove our main theorem and consider our definition (Definition 1.2) of elements of the dual $A^{*}$ of rank at most $k$ and singleton support in order to relate it to familiar concepts of extreme points of the unit ball of $A^{*}$, pure states and factorial states on $A$.

Proof of Theorem 1.3. Now let $T: A \rightarrow A$ be an elementary operator of the form

$$
T x=\sum_{j=1}^{\ell} a_{j} x b_{j} \quad\left(a_{j}, b_{j} \in M(A)\right) .
$$


If $T$ is a $k$-positive elementary operator on $A$ and $\pi_{t}$ is an irreducible representation of $A$, then the elementary operator $T_{\pi_{t}}$

$$
x \mapsto \sum_{j=1}^{\ell} \pi_{t}\left(a_{j}\right) x \pi_{t}\left(b_{j}\right): \mathcal{B}\left(H_{t}\right) \rightarrow \mathcal{B}\left(H_{t}\right)
$$

must be $k$-positive. Conversely, if all of these operators are $k$-positive, then $T$ must be $k$-positive (which can be seen by considering the normal extension $T_{a}$ of $T$ to $\mathcal{A}$ ).

Hence the result follows immediately from Theorem 1.1 and the definitions of $Q_{T}$ and $\mathcal{F}_{k}^{s}(A)$.

Example 4.1. Each pure state $\phi$ on $A$ is an element of $\mathcal{F}_{1}^{s}(A)$. So is every multiple of a pure state.

This follows because the irreducible representation $\pi_{\phi}: A \rightarrow \mathcal{B}\left(H_{\phi}\right)$ is cyclic and the rank-one projection onto the cyclic vector is easily seen to give rise to $\phi$ via the trace duality.

Example 4.2. Every extreme point of the unit ball of $A^{*}$ is in $\mathcal{F}_{1}^{s}(A)$.

In [2, Proposition 1.1] it is explained in detail (using [1, Proposition 2.1]) that every such extreme point can be represented as $\phi(x)=\langle\pi(x) \xi, \eta\rangle$ for an irreducible representation $\pi: A \rightarrow \mathcal{B}(H)$ and unit vectors $\xi, \eta \in H$. In other words, $\phi(x)=\operatorname{tr}\left(\pi(x)\left(\eta^{*} \otimes \xi\right)\right)$ and so $\phi \in \mathcal{F}_{1}^{s}(A)$.

Example 4.3. For $A=\mathcal{K}(H)$ the only irreducible representation of $A$ (up to equivalence) is the inclusion $A \subset \mathcal{B}(H)$ and so $\mathcal{F}_{k}^{s}(A)$ can be identified with the elements of $\mathcal{F}_{k}(H)$ via the trace duality.

Example 4.4. For $A=\mathcal{B}(H)$ one irreducible representation of $A$ is the identity and we can identify $\mathcal{F}_{k}(H)$ with a subset of $\mathcal{F}_{k}^{s}(A)$.

Lemma 4.5. Let $A$ be a $C^{*}$-algebra. For $k \geqslant 1, \phi \in \mathcal{F}_{k}^{s}(A)$ if and only if $\phi \in A^{*}$ and the absolute value $|\phi|$ is a non-negative multiple of some $\psi \in F_{k}(A)$.

Proof. The polar decomposition (as in [12]) for a normal state $\phi$ on a von-Neumann algebra $\mathcal{M}$ states that $|\phi|$ satisfies $\phi=v|\phi|$ for a partial isometry $v \in \mathcal{M}$ (or $\phi(x)=$ $|\phi|(x v)$ for $x \in \mathcal{M})$. We have that $v^{*} v$ is the support of $|\phi|$. For $\phi \in A^{*}$, we consider $|\phi|$ relative to the normal extension of $\phi$ to $A^{* *}$.

Take $\phi \in \mathcal{F}_{k}^{S}(A)$ and we leave aside the trivial case $\phi=0$. Write $\phi(x)=\operatorname{tr}\left(\pi_{t}(x) y\right)$, where $y \in \mathcal{F}_{k}\left(H_{t}\right)$. Via polar decomposition, we can express $y=v|y|$ for some partial isometry $v$ of $H_{t}$ (and both $|y|$ and $v$ have the same rank as $y$ ). Then it is easy to see from a diagonalization of $|y|$ that $x \mapsto \operatorname{tr}\left(\pi_{t}(x)|y|\right)$ is a multiple $\lambda \psi$ of a state $\psi \in$ $F_{k}(A)$, with $\lambda \geqslant 0$ the sum of the eigenvalues of $|y| . \lambda$ is then the trace class norm of $y\left(=\|\phi\|\right.$, as can be seen by considering the norm of the extension of $\phi$ to $\left.A^{* *}\right)$ and $\psi(x)=\operatorname{tr}\left(\pi_{t}(x)(|y| / \lambda)\right)$. Also $\phi(x)=\lambda \psi(x v)$ for $x \in A$ and this extends to $x \in A^{* *}$. From the Cauchy-Schwarz inequality for $\psi$ it follows that $|\phi(x)|^{2} \leqslant \lambda^{2} \psi\left(x^{*} x\right)$ and then it follows from [12, Proposition III.4.6] that $|\phi|=\lambda \psi$. 
Conversely, we know $F_{k}(A) \subset \mathcal{F}_{k}^{s}(A)$ and so any $\psi \in F_{k}(A)$ can be expressed as $\psi(x)=\operatorname{tr}\left(\pi_{t}(x) y\right)$ for some $y \in \mathcal{F}_{k}\left(H_{t}\right)$, some $t \in \hat{A}$. If $v \in A^{* *}$ is the partial isometry with $v^{*} v$ the support of $\psi$ and $\phi(x)=|\phi|(x v)=\lambda \psi(x v)$ for $x \in A^{* *}$ (some $\lambda>0$ ), then we claim that $v$ must be in $\pi_{t}(A)^{\prime \prime} \equiv \mathcal{B}\left(H_{t}\right)$. To check the claim, consider the central projection $z \in A^{* *}$ such that $\pi_{t}(A)^{\prime \prime}=z A^{* *}$. Then $w=z v$ is a partial isometry with the same properties as $v\left(w^{*} w=z v^{*} v=v^{*} v, \lambda \psi(x w)=\lambda \psi(x v)\right)$ and by the uniqueness of polar decompositions, $w=v$. It follows that $\lambda \psi(x v)=\lambda \operatorname{tr}\left(\pi_{t}(x) \pi_{t}^{\prime \prime}(v) y\right)$ and $\pi_{t}^{\prime \prime}(v) y \in \mathcal{F}_{k}\left(H_{t}\right)$. Thus $x \mapsto \lambda \psi(x v)$ is in $\mathcal{F}_{k}^{s}(A)$.

Proof of Theorem 1.4. If $T$ is $k$-positive, then

$$
x \mapsto T\left(a^{*} x a\right)=\sum_{j=1}^{\ell}\left(a_{j} a^{*}\right) x\left(a b_{j}\right)
$$

must also be $k$-positive for each $a \in A$. Since $F_{k}(A) \subset \mathcal{F}_{k}^{s}(A)$, applying Theorem 1.3 to this new $k$-positive elementary operator gives the desired inequality.

Conversely, assume the inequality for all $\psi \in F_{k}(A)$ and $a \in A$. Now take $\phi \in \mathcal{F}_{k}^{s}(A)$, which can be represented as

$$
\phi(x)=\operatorname{tr}\left(\pi_{t}(x) \sum_{j=1}^{k^{\prime}} \eta_{j}^{*} \otimes \xi_{j}\right)=\sum_{j=1}^{k^{\prime}}\left\langle\pi_{t}(x) \xi_{j}, \eta_{j}\right\rangle
$$

with $t \in \hat{A}, k^{\prime} \leqslant k, \xi_{j}, \eta_{j} \in H_{t}\left(1 \leqslant j \leqslant k^{\prime}\right)$. We can further assume that the $\xi_{j}$ are orthonormal. By the transitivity theorem, there exists $\tilde{a} \in A$ with $\eta_{j}=\pi_{t}(\tilde{a}) \xi_{j}$ for $1 \leqslant j \leqslant k^{\prime}$ and then

$$
\phi(x)=\sum_{j=1}^{k^{\prime}}\left\langle\pi_{t}\left(\tilde{a}^{*} x\right) \xi_{j}, \xi_{j}\right\rangle=\psi\left(k^{\prime} \tilde{a}^{*} x\right)=\psi(a x)
$$

if we take $\psi \in F_{k}(A)$ to be given by $\psi(x)=\left(1 / k^{\prime}\right) \sum_{j=1}^{k^{\prime}}\left\langle\pi_{t}(x) \xi_{j}, \xi_{j}\right\rangle$ and $a=k^{\prime} \tilde{a}^{*}$. Observe then that

$$
\phi^{*}(x)=\overline{\phi\left(x^{*}\right)}=\overline{\psi\left(a x^{*}\right)}=\psi\left(x a^{*}\right) .
$$

Thus, from (1.1) we can apply Theorem 1.3 to show that $T$ is $k$-positive.

(Our original argument for the converse used Lemma 4.5. This more direct argument was pointed out to the author by Archbold, whom we thank for permission to include it.)

Corollary 4.6. For a $C^{*}$-algebra $A$ and $k \geqslant 1$, the following conditions are equivalent.

(i) $A$ is either $k$-subhomogeneous or an antiliminal extension of a $k$-subhomogeneous $C^{*}$-algebra.

(ii) $F_{k}(A)$ is weak ${ }^{*}$-dense in $F_{k+1}(A)$.

(iii) $F_{k}(A)$ is weak ${ }^{*}$-dense in $F_{m}(A)$ for each $m \geqslant k$. 
(iv) For each $m \geqslant k, F_{m}(A)$ is contained in the weak*-closure of the intersection of the unit ball of $A^{*}$ with $\mathcal{F}_{k}^{s}(A)$.

(v) For each $m \geqslant k, F_{m}(A)$ is contained in the weak*-closure of $\mathcal{F}_{k}^{s}(A)$.

(vi) Every $k$-positive elementary operator on $A$ is completely positive.

(vii) $F_{k+1}(A)$ is contained in the weak ${ }^{*}$-closure of $\mathcal{F}_{k}^{s}(A)$.

(viii) Every $k$-positive elementary operator on $A$ of the form $T x=\sum_{j=1}^{\ell} a_{j} x b_{j}\left(a_{j}, b_{j} \in A\right.$ for $1 \leqslant j \leqslant \ell)$ is $(k+1)$-positive.

Proof. The equivalence of (i)-(iii) is shown in [5]. Condition (i) means that the (unique) maximal postliminal ideal $J \subset A$ is a $k$-subhomogeneous $C^{*}$-algebra. The implications (iii) $\Rightarrow$ (iv) $\Rightarrow$ (v) are obvious.

$(\mathrm{v}) \Rightarrow(\mathrm{vi})$. Suppose $T(x)=\sum_{j=1}^{\ell} a_{j} x b_{j}$ is a $k$-positive elementary operator on a $C^{*}$ algebra where (v) holds. Then using Theorem 1.4, it is sufficient to show that (1.1) holds whenever $\psi \in F_{m}(A), m>k$ and $a \in A$. Fixing such a $\psi$ and $a$, we can find a net $\left(\phi_{\alpha}\right)_{\alpha}$ in $\mathcal{F}_{k}^{S}(A)$ which converges weak* to $\psi$, so that $\phi_{\alpha}(a x) \rightarrow \psi(a x)$ for each $x \in M(A)$. (Note that $a x \in A$.) Now, $\phi_{\alpha} a \in \mathcal{F}_{k}^{s}(A)$, where $\left(\phi_{\alpha} a\right)(x)=\phi_{\alpha}(a x)$ and

$$
\left(\phi_{\alpha} a\right)^{*}(x)=\overline{\left(\phi_{\alpha} a\right)\left(x^{*}\right)}=\overline{\phi_{\alpha}\left(a x^{*}\right)} \rightarrow \overline{\psi\left(a x^{*}\right)}=\psi\left(x a^{*}\right)
$$

for $x \in A$. Since $\phi_{\alpha} a \in \mathcal{F}_{k}^{s}(A)$ we can apply Theorem 1.3 to get $Q_{T}\left(\phi_{\alpha} a\right) \geqslant 0$ or

$$
\sum_{j=1}^{\ell}\left(\phi_{\alpha} a\right)^{*}\left(a_{j}\right)\left(\phi_{\alpha} a\right)\left(b_{j}\right) \geqslant 0 .
$$

Taking a limit, we get (1.1).

(vi) $\Rightarrow(\mathrm{v})$. Assume $\psi \in F_{m}(A)(m>k)$ is not in the weak*-closure of $\mathcal{F}_{k}^{s}(A)$. Then there exist $a_{1}, a_{2}, \ldots, a_{\ell} \in A$ such that

$$
\max _{1 \leqslant j \leqslant \ell}\left|\psi\left(a_{j}\right)-\phi\left(a_{j}\right)\right|>1
$$

for each $\phi \in \mathcal{F}_{k}^{s}(A)$. Define $\gamma(\phi) \in \mathbb{C}^{\ell}$ by $\gamma(\phi)=\left(\phi\left(a_{1}\right), \phi\left(a_{2}\right), \ldots, \phi\left(a_{\ell}\right)\right)$ and similarly define $\gamma(\psi)$. Then it follows that $\left\{\gamma(\phi): \phi \in \mathcal{F}_{k}^{s}(A)\right\}$ is a 'cone' in $\mathbb{C}^{\ell}$ (closed under multiplication by complex scalars) and, moreover, the (Euclidean) distance from $\gamma(\psi)$ to this cone is at least 1. It follows that there is an upper bound $c=\sqrt{\|\gamma(\psi)\|^{2}-1} /\|\gamma(\psi)\|<1$ on the cosine of the angle in $\mathbb{C}^{\ell}$ between $\gamma(\psi)$ and this cone. Hence

$$
|\langle\gamma(\psi), \gamma(\phi)\rangle| \leqslant c\|\gamma(\psi)\|\|\gamma(\phi)\|
$$

for each $\phi \in \mathcal{F}_{k}^{s}(A)$. Let $\left(\lambda_{1}, \lambda_{2}, \ldots, \lambda_{\ell}\right)=\overline{\gamma(\psi)} /(c\|\gamma(\psi)\|)$ and consider the elementary operator $T: A \rightarrow A$ with

$$
T x=\sum_{j=1}^{\ell} a_{j}^{*} x a_{j}-\left(\sum_{j=1}^{\ell} \lambda_{j} a_{j}\right)^{*} x\left(\sum_{j=1}^{\ell} \lambda_{j} a_{j}\right) .
$$


It follows by Theorem 1.3 , that $T$ is $k$-positive because

$$
Q_{T}(\phi)=\sum_{j=1}^{\ell}\left|\phi\left(a_{j}\right)\right|^{2}-\left|\sum_{j=1}^{\ell} \phi\left(a_{j}\right) \lambda_{j}\right|^{2}=\|\gamma(\phi)\|^{2}-\frac{|\langle\gamma(\phi), \gamma(\psi)\rangle|^{2}}{c^{2}\|\gamma(\psi)\|^{2}} \geqslant 0,
$$

but $T$ is not $m$-positive because $Q_{T}(\psi)<0$.

(vii) $\Longleftrightarrow$ (viii) can be proved by the same argument (since the case $a_{j}, b_{j} \in M(A)$ is not needed for (vi) $\Rightarrow(\mathrm{v})$ ) and clearly (vi) implies (viii).

To prove that (viii) implies (i) and complete the proof, observe that condition (viii) implies that the same is true of each (closed two-sided) ideal $J \subset A$. Each $k$-positive elementary $T: J \rightarrow J$ of the form given in (viii) $\left(a_{j}, b_{j} \in J\right.$ for each $j$ ) gives rise to a $k$-positive elementary $T_{1}$ on $A$ via $T_{1} x=\sum_{j=1}^{\ell} a_{j} x b_{j}$ (see [3, Lemma 2(i)]). Hence $T_{1}$ is $(k+1)$-positive and it follows that $T$ is $(k+1)$-positive.

Taking $J$ to be the maximal postliminal ideal of $A$, our goal is to show that $J$ is $k$-subhomogeneous. For this we rely on the following lemma.

Lemma 4.7. Suppose $A$ is a continuous trace $C^{*}$-algebra which satisfies condition (viii) of Corollary 4.6. Then $A$ is $k$-subhomogeneous.

Proof. We have proved that (viii) $\Rightarrow$ (vii) in Corollary 4.6 and so we can conclude that $F_{k+1}(A)$ is contained in the closure of $\mathcal{F}_{k}^{s}(A)$ in the present case.

If $A$ is not $k$-subhomogeneous, there exists $t \in \hat{A}$ so that the associated representation $\pi_{t}: A \rightarrow \mathcal{B}\left(H_{t}\right)$ has $H_{t}$ of dimension at least $k+1$. Choose $k+1$ non-zero orthogonal $\xi_{j} \in H_{t}$, normalized so that $\sum_{j=1}^{k+1}\left\|\xi_{j}\right\|^{2}=1$, and put $y=\sum_{j=1}^{k+1} \xi_{j}^{*} \otimes \xi_{j}$. Then we can define $\psi \in F_{k+1}(A) \backslash F_{k}(A)$ by $\psi(a)=\operatorname{tr}\left(\pi_{t}(a) y\right)$.

Since $A$ must obey Fell's condition, there is a $p \in A$ such that $\pi_{s}(p)$ is a rank-one projection for all $s$ in some neighbourhood of $t$ in $\hat{A}$. Using the Dauns-Hoffman theorem, we can find $q \in A$ so that $\pi_{s}(q)=\theta_{s}^{*} \otimes \theta_{s}$ is a positive element of $\mathcal{B}\left(H_{s}\right)$ of rank at most one for all $s \in \hat{A}$ and $\pi_{t}(q)=\pi_{t}(p)$. By the transitivity theorem, there exist $a_{j} \in A$ $(1 \leqslant j \leqslant k+1)$ so that $\pi_{t}\left(a_{j}\right) \theta_{t}=\xi_{j}$. Note that

$$
\begin{aligned}
\xi_{i}^{*} \otimes \xi_{j} & =\left(\pi_{t}\left(a_{i}\right) \theta_{t}\right)^{*} \otimes\left(\pi_{t}\left(a_{j}\right) \theta_{t}\right) \\
& =\pi_{t}\left(a_{j}\right)\left(\theta_{t}^{*} \otimes \theta_{t}\right) \pi_{t}\left(a_{i}^{*}\right) \\
& =\pi_{t}\left(a_{j}\right) \pi_{t}(q) \pi_{t}\left(a_{i}^{*}\right)=\pi_{t}\left(a_{j} q a_{i}^{*}\right) .
\end{aligned}
$$

By a short computation, we can compute $y_{i j}=\psi\left(a_{j} q a_{i}^{*}\right)$ and get $y_{i j}=\delta_{i j}$. In particular the $(k+1) \times(k+1)$ matrix with entries $y_{i j}$ has determinant 1 .

Setting $\eta_{s, j}=\pi_{s}\left(a_{j}\right) \theta_{s} \in H_{s}$, we get

$$
\eta_{s, i}^{*} \otimes \eta_{s, j}=\pi_{s}\left(a_{j} q a_{i}^{*}\right) .
$$

If $\phi_{1} \in \mathcal{F}_{1}^{S}(A)$, then the $(k+1) \times(k+1)$ matrix with entries $\phi_{1}\left(a_{j} q a_{i}^{*}\right)$ has rank at most one. To verify this, suppose the support of $\phi_{1}$ is $s$ and then $\phi_{1}$ can be represented as $\phi_{1}(a)=\operatorname{tr}\left(\pi_{s}(a)\left(\theta^{*} \otimes \mu\right)\right)$ for $\theta, \mu \in H_{s}$. Then we can compute

$$
\phi_{1}\left(a_{j} q a_{i}^{*}\right)=\operatorname{tr}\left(\left(\eta_{s, i}^{*} \otimes \eta_{s, j}\right)\left(\theta^{*} \otimes \mu\right)\right)=\left\langle\eta_{s, j}, \theta\right\rangle\left\langle\mu, \eta_{s, i}\right\rangle .
$$


It follows that if $\phi \in \mathcal{F}_{k}^{s}(A)$, then the matrix with entries $\phi\left(a_{j} q a_{i}^{*}\right)$ has rank at most $k$ and determinant zero. As this is true for all $\phi \in \mathcal{F}_{k}^{s}(A)$ and the $\operatorname{determinant} \operatorname{det}\left(\phi\left(a_{j} q a_{i}^{*}\right)\right)_{i, j=1}^{k+1}$ is a continuous function of $\phi$, the closure of $\mathcal{F}_{k}^{s}(A)$ cannot include $\psi$.

This contradiction shows that $A$ must be $k$-subhomogeneous.

We now complete the proof of Corollary 4.6. Recall that $J$ denotes the maximal postliminal ideal of $A$. The set ${ }_{k} \hat{J}$ of those $t \in \hat{J}$ such that the associated representation $\pi_{t}$ has rank at most $k$ is closed in $\hat{J}$ (see $[\mathbf{9}, 4.4 .10,6.1 .5]$ ). There is an essential continuous trace ideal $I_{\mathrm{c}} \subset J[\mathbf{9}, 6.2 .11]$ and $\hat{I}_{\mathrm{c}}$ can be identified with a dense subset of $\hat{J}$. As observed already, $I_{\mathrm{c}}$ inherits condition (viii) from $J$. By the lemma, $\hat{I}_{\mathrm{c}} \subset{ }_{k} \hat{J}$. Hence ${ }_{k} \hat{J}=\hat{J}$.

As noted in $\S 1$, the case $k=1$ is dealt with in [3] where an if and only if result is established between (i) and (vi).

Acknowledgements. The author thanks Wend Werner for helpful conversations in connection with this work and for pointing out an error in an earlier version. He also thanks R. Archbold and D. O'Donovan for helpful remarks.

\section{References}

1. C. A. Akemann and B. Russo, Geometry of the unit sphere of a $C^{*}$-algebra and its dual, Pac. J. Math. 32 (1970), 575-585.

2. R. J. Archbold And F. W. Shultz, Characterization of $C^{*}$-algebras with continuous trace by properties of their pure states, Pac. J. Math. 136 (1989), 1-13.

3. R. J. Archbold, M. Mathieu and D. W. B. Somerset, Elementary operators on antiliminal $C^{*}$-algebras, Math. Annln 313 (1999), 609-616.

4. W. Arveson, The index of a quantum dynamical semigroup, J. Funct. Analysis 146 (1997), 557-588.

5. C. J. K. Batty and R. J. ARchbold, On factorial states of operator algebras, II, $J$. Operat. Theory 13 (1985), 131-142.

6. M. D. ChoI, Positive linear maps on $C^{*}$-algebras, Can. J. Math. 24 (1972), 520-529.

7. M. Mathieu, Elementary operators on prime $C^{*}$-algebras, I, Math. Annln 284 (1989), 223-244.

8. V. I. PAulsen, Completely bounded maps and dilations, Pitman Notes in Mathematics Series, vol. 146 (Pitman, New York, 1986).

9. G. K. Pedersen, $C^{*}$-algebras and their automorphism groups (Academic Press, London, 1979).

10. W. F. Stinespring, Positive functions on $C^{*}$-algebras, Proc. Am. Math. Soc. 6 (1955), 211-216.

11. T. TAKASAKi And J. TOMiYama, On the geometry of positive maps in matrix algebras, Math. Z. 184 (1983), 101-108.

12. M. TAKESAKI, Theory of operator algebras, vol. I (Springer, 1979).

13. R. M. Timoney, A note on positivity of elementary operators, Bull. Lond. Math. Soc. 32 (2000), 229-234. 